Pacific

Journal of

Mathematics

TRANSITION DENSITY ESTIMATES FOR STABLE PROCESSES ON SYMMETRIC SPACES

PIOTR GRACZYK

Volume $217 \quad$ No. 1

November 2004 


\title{
TRANSITION DENSITY ESTIMATES FOR STABLE PROCESSES ON SYMMETRIC SPACES
}

\author{
PiOTR GRACZYK
}

\begin{abstract}
We present estimates of the transition densities for stable processes on Riemannian symmetric spaces of noncompact type. We show that these processes have a weak scaling property and we address in this way a question of Getoor about the stability properties of pseudostable measures on symmetric spaces.
\end{abstract}

\section{Introduction}

Stable Lévy processes on a group, where stability is meant with respect to group automorphisms, can only exist on nilpotent groups (see $[\mathbf{K u}],[\mathbf{A p p} \mathbf{1}]$, [App2]). In [Ge], using a subordination procedure, Getoor defined stable processes with respect to Brownian motion on hyperbolic spaces of noncompact type. He asked whether stability properties of such a process and its semigroup can be found. In this paper we answer this question positively (see Theorem 4.3 and Remark 1 thereafter), proving a weak scaling property of the transition densities of the stable process in the sense of Getoor. We also solve in this way, in the case of symmetric spaces, Open Problem 4 from [App2]. The main results of our paper are given in Theorem 4.3 and Corollaries 5.3 and 5.6.

\section{Preliminaries}

Let $G$ denote a noncompact semisimple Lie group, $K$ a maximal compact subgroup, and $X=G / K$ the associated Riemannian symmetric space with nonpositive curvature. We adopt the notation and conventions from $[\mathbf{A J}]$. In particular, if $\mathfrak{a}$ is the Cartan space and $\lambda \in \mathfrak{a}$, then we denote by $\phi_{\lambda}$ the spherical functions on $X$.

It is well-known that the heat kernel on $X=G / K$ is given by

$$
h_{t}(x)=C \int_{\mathfrak{a}} \frac{d \lambda}{|c(\lambda)|^{2}} e^{-t\left(|\lambda|^{2}+|\rho|^{2}\right)} \phi_{\lambda}(x)
$$

where $C=C(X)$ is a constant, $c(\lambda)$ is the $c$-function appearing in the inverse spherical Fourier transform formula and $\rho=\frac{1}{2} \sum_{\alpha>0} m_{\alpha} \alpha$ is the half-sum of the positive roots with multiplicities $m_{\alpha}$. 
We set $n=\operatorname{dim} X, m=\sum_{\alpha>0} m_{\alpha}$, and we denote by $\Sigma^{++}$the set of positive indivisible roots and by $\mathfrak{a}^{+} \subset \mathfrak{a}$ the positive Weyl chamber. We have global estimates:

Theorem 2.1 ([AJ], Theorem 3.7). Let $k>0$. Then

(1) $h_{t}(\exp H) \asymp t^{-\frac{n}{2}}(1+t)^{\frac{m}{2}-\left|\Sigma^{++}\right|}\left(\prod_{\alpha \in \Sigma^{++}}(1+\langle\alpha, H\rangle)\right) e^{-|\rho|^{2} t-\langle\rho, H\rangle-\frac{|H|^{2}}{4 t}}$, provided $|H|<k(1+t), H \in \overline{\mathfrak{a}^{+}}$.

Observe that $\left(\prod_{\alpha \in \Sigma^{++}}(1+\langle\alpha, H\rangle)\right) e^{-\langle\rho, H\rangle} \asymp \phi_{0}(\exp H)$ when $H \in \overline{\mathfrak{a}^{+}}$. Thus for any $x \in X$ we have

$$
h_{t}(x) \asymp t^{-\frac{n}{2}}(1+t)^{\frac{m}{2}-\left|\Sigma^{++}\right|} \phi_{0}(x) e^{-|\rho|^{2} t-\frac{|x|^{2}}{4 t}},
$$

where $|x|=d\left(x_{0}, x\right)$, the Riemannian distance between $x \in X$ and $x_{0}=e K$.

From the probabilistic point of view, the $h_{t}(x)$ are the densities of the Brownian motion on $X=G / K$. If $\left(X_{t}\right)$ is the corresponding diffusion on $G$ with stationary independent (left) increments and the distribution of $X_{0}$ given by the Haar measure on $K$ then its transition function is

$$
P_{t}(x, y)=h_{t}\left(x^{-1} y\right), \quad x, y \in G .
$$

Convention: by $c$ without subscripts we denote a positive constant that may vary from term to term, but otherwise depends only on the underlying space and $\alpha$ (see below).

\section{Stable semigroups}

In the sequel we understand the objects under discussion (processes, transition probabilities, etc.) equivalently on the symmetric space $X=G / K$ or on the group $G$, without changing notation.

Let $\alpha \in(0,2)$. The $\alpha$-stable process on a symmetric space $X$ with transition densities $P_{t}(x, y)=p_{t}\left(x^{-1} y\right)$ was defined by Getoor $([\mathbf{G e}])$ by means of a subordination procedure. In particular,

$$
p_{t}(x)=\int_{0}^{\infty} h_{u}(x) \eta_{t}(u) d u,
$$

where $\eta_{t}$ is the density of the $\alpha / 2$-stable subordinator (cf. also [Be]).

In $[\mathbf{A J}]$ estimates for $p_{t}$ when $\alpha=1$ were given. In [Gruet] estimates for $p_{t}(x)$ when $t \rightarrow \infty$ were given in the rank-one case. In this paper we obtain estimates for $p_{t}(x)$ with respect to both variables $x, t$ for any $\alpha \in(0,2)$ on all Riemannian symmetric spaces of noncompact type.

It is well-known that the symmetric stable densities on Euclidean spaces cannot be written explicitly, except for $\alpha=1$. We now recall the exact estimates of the densities $\eta_{t}(u)$, which will be crucial for our estimates of $p_{t}(x)$. 
By Theorem 37.1 of $[\mathbf{D}]$,

$$
\lim _{u \rightarrow \infty} \eta_{1}(u) u^{1+\alpha / 2}=\frac{\alpha}{2 \Gamma\left(1-\frac{\alpha}{2}\right)} .
$$

This, together with the boundedness of $\eta_{1}(\cdot)$ and the scaling property

$$
\eta_{t}(u)=t^{-2 / \alpha} \eta_{1}\left(t^{-2 / \alpha} u\right), \quad t, u>0
$$

gives

$$
\begin{array}{ll}
\eta_{t}(u) \leq c t u^{-1-\alpha / 2}, & t, u>0, \\
\eta_{t}(u) \geq \tilde{c} t u^{-1-\alpha / 2}, & t>0, u>t^{2 / \alpha} .
\end{array}
$$

Moreover, by $[\mathbf{H}]$ and (5) we know that

$$
\eta_{t}(u) \asymp t^{\frac{1}{2-\alpha}} u^{-\frac{4-\alpha}{4-2 \alpha}} e^{-c_{1} t^{\frac{2}{2-\alpha}} u^{-\frac{\alpha}{2-\alpha}}}, \quad t^{-2 / \alpha} u \in(0,1),
$$

where $c_{1}=c_{1}(\alpha)=\frac{2-\alpha}{2}\left(\frac{\alpha}{2}\right)^{\frac{\alpha}{2-\alpha}}$. Observe that for $u>t^{2 / \alpha}$ we have

$$
\exp \left(-c_{1} t^{\frac{2}{2-\alpha}} u^{-\frac{\alpha}{2-\alpha}}\right) \geq \exp \left(-c_{1}\right)
$$

so that (6) and (7) give

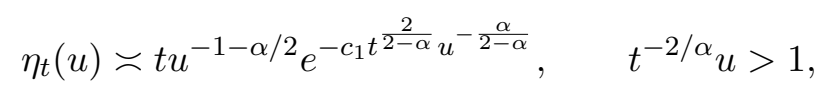

which of course simplifies to $\eta_{t}(u) \asymp t u^{-1-\alpha / 2}$, but we want to make the estimates (8) and (9) as similar as possible.

Consider now the case of an $n$-dimensional Riemannian symmetric space $X=G / K$ when $G$ is a complex Lie group. We have then

$$
h_{u}(x)=\phi_{0}(x)(4 \pi u)^{-n / 2} e^{-\frac{|x|^{2}}{4 u}-|\rho|^{2} u}
$$

and $h_{u}(\exp H)$ is a probability density with respect to the polar coordinate Jacobian $J(H) d H=\operatorname{vol}(K / M) \prod_{\alpha \in \Sigma^{+}} \sinh ^{m_{\alpha}} \alpha(H) d H$. Thanks to this explicit formula for $h_{u}$ we are able to compute the 1-stable density in the complex case, in terms of the modified Bessel function of the third kind (or the MacDonald function) $K_{\nu}(x)$.

Proposition 3.1. If $G$ is a complex Lie group and $\alpha=1$ then

$$
p_{t}(x)=2\left(\frac{2 \pi}{|\rho|}\right)^{-\frac{n+1}{2}} t \phi_{0}(x)\left(|x|^{2}+t^{2}\right)^{-\frac{n+1}{4}} K_{\frac{n+1}{2}}\left(|\rho| \sqrt{|x|^{2}+t^{2}}\right) .
$$

Proof. For $\alpha=1$ we have $\eta_{t}(u)=\frac{t}{2 \sqrt{\pi}} u^{-3 / 2} \exp \left(-\frac{t^{2}}{4 u}\right)$. By using the subordination formula and an integral representation for MacDonald function [GR], p. 907, (8.432.6),

$$
K_{\nu}(z)=\frac{1}{2}\left(\frac{z}{2}\right)^{\nu} \int_{0}^{\infty} e^{-t-\frac{z^{2}}{4 t}} t^{-\nu-1} d t,
$$

we get the desired assertion. 


\section{Estimates of $p_{t}(x)$}

By (8) and (9) we have

$$
\begin{aligned}
& p_{t}(x) \asymp t^{\frac{1}{2-\alpha}} \int_{0}^{t^{2 / \alpha}} h_{u}(x) u^{-\frac{4-\alpha}{4-2 \alpha}} e^{-c_{1} t^{\frac{2}{2-\alpha}} u^{-\frac{\alpha}{2-\alpha}} d u} \\
& \quad+t \int_{t^{2 / \alpha}}^{\infty} h_{u}(x) u^{-1-\alpha / 2} e^{-c_{1} t^{\frac{2}{2-\alpha}} u^{-\frac{\alpha}{2-\alpha}}} d u .
\end{aligned}
$$

As in $[\mathbf{A J}]$ we will see that the main contribution in (10) will come from the interval where

$$
u \sim u_{0}=u_{0}(x, t)
$$

with $u_{0}$ minimizing the function

$$
f(u)=\frac{|x|^{2}}{4 u}+|\rho|^{2} u+c_{1} t^{\frac{2}{2-\alpha}} u^{-\frac{\alpha}{2-\alpha}} .
$$

The exponent equal to $-f$ is then maximal in the exponential appearing in (10), if we replace $h_{u}(x)$ by its estimate given in Theorem 2.1. More precisely, when $|x|<k(1+u)$ and $u>\delta>0$ with $\delta$ fixed, we have

$$
h_{u}(x) \eta_{t}(u) \asymp \phi_{0}(x) G(t, u) e^{-\frac{|x|^{2}}{4 u}-|\rho|^{2} u-c_{1} t^{2-\alpha}} u^{-\frac{\alpha}{2-\alpha}},
$$

where

$$
G(t, u)= \begin{cases}t^{\frac{1}{2-\alpha}} u^{-r(\alpha)}, & u \leqslant t^{2 / \alpha}, \\ t u^{-s(\alpha)}, & u \geqslant t^{2 / \alpha},\end{cases}
$$

with $r(\alpha)=\frac{l}{2}+\left|\Sigma^{++}\right|+\frac{4-\alpha}{4-2 \alpha}$ and $s(\alpha)=\frac{l}{2}+\left|\Sigma^{++}\right|+1+\frac{\alpha}{2}$, where $l=\operatorname{dim} \mathfrak{a}$ is the rank of $X$ (we have used $l=n-m$ ).

Since

$$
f^{\prime}(u)=-\frac{|x|^{2}}{4 u^{2}}+|\rho|^{2}-\frac{c_{1} \alpha}{2-\alpha} \frac{t^{\frac{2}{2-\alpha}}}{u^{\frac{2}{2-\alpha}}}
$$

we get

$$
|\rho|^{2} u_{0}^{2}=\frac{|x|^{2}}{4}+\frac{c_{1} \alpha}{2-\alpha} t^{\frac{2}{2-\alpha}} u_{0}^{\frac{2(1-\alpha)}{2-\alpha}}
$$

or, in more convenient form,

$$
|\rho|^{2}=\frac{1}{4}\left(\frac{|x|}{u_{0}}\right)^{2}+c_{2}\left(\frac{t}{u_{0}}\right)^{\frac{2}{2-\alpha}},
$$

with $c_{2}=c_{1} \alpha /(2-\alpha)$.

Remark. Equation (13) can be solved explicitly as a (bi)quadratic equation for $\frac{2(1-\alpha)}{2-\alpha}=-2,0,1,2,4$, corresponding respectively to $\alpha=\frac{3}{2}, 1,0, \emptyset, 3$, so only for $\alpha=\frac{3}{2}$ or 1 . In the case $\alpha=1$ we obtain $|\rho|^{2} u_{0}^{2}=\frac{|x|^{2}}{4}+c_{1} t^{2}=$ 
$\frac{|x|^{2}+t^{2}}{4}$ and $u_{0}=\sqrt{|x|^{2}+t^{2}} / 2|\rho|$ as in $[\mathbf{A J}]$. In the case $\alpha=\frac{3}{2}$ we obtain a biquadratic equation and

$$
u_{0}=\frac{1}{|\rho| \sqrt{2}}\left(\frac{|x|^{2}}{4}+\sqrt{\frac{|x|^{4}}{16}+4 c_{2} t^{4}|\rho|^{2}}\right)^{\frac{1}{2}} .
$$

Equation (13) may be solved explicitly for some other particular values of $\alpha$ (see the following lemma). For a generic $\alpha \in(0,2)$ the function $u_{0}$ is given only implicitly by (13).

Lemma 4.1. For $\alpha=4 / 3$ and $t>c_{3}|x|$ with $c_{3}=\frac{1}{\sqrt{3}\left(4 c_{2}|\rho|\right)^{1 / 3}}$, we have $u_{0}=\frac{1}{\sqrt[3]{2|\rho|^{2}}}\left(\left(c_{2} t^{3}-\sqrt{c_{2}^{2} t^{6}-\frac{|x|^{6}}{432|\rho|^{2}}}\right)^{\frac{1}{3}}+\left(c_{2} t^{3}+\sqrt{c_{2}^{2} t^{6}-\frac{|x|^{6}}{432|\rho|^{2}}}\right)^{\frac{1}{3}}\right)$.

Proof. If $\alpha=\frac{4}{3}$ then (13) becomes by rearrangement an equation of degree 3 with respect to $u_{0}$. It can be solved explicitly by Cardano formulas. However, a reasonable expression occurs only when the determinant is positive. This is ensured by the second condition in the hypothesis.

Lemma 4.2. For each $x$ and there exists exactly one solution $u_{0}(x, t)$ of (13) and (14). We have $u_{0} \asymp|x|+t$. The functions $(H, t) \rightarrow u_{0}(\exp H, t)$ and $(H, t) \rightarrow f\left(u_{0}(\exp H, t)\right)$ are homogeneous of degree 1 .

Proof. From (14) we have $\left(|x| / u_{0}\right)^{2}<4|\rho|^{2}$ and $\left(t / u_{0}\right)^{2 /(2-\alpha)}<c_{2}^{-1}|\rho|^{2}$, so that $u_{0}>|x| /(2|\rho|)$ and $u_{0}>t c_{2}^{(2-\alpha) / 2}|\rho|^{-(2-\alpha)}$, which gives the lower bound. On the other hand, for any constant $A>1$ we have

$$
\frac{1}{4}\left(\frac{|x|}{A(|x|+t)}\right)^{2}+c_{2}\left(\frac{t}{A(|x|+t)}\right)^{\frac{2}{2-\alpha}} \leq \frac{1}{4 A^{2}}+c_{2}\left(\frac{1}{A}\right)^{\frac{2}{2-\alpha}},
$$

which is less than $|\rho|^{2}$ for $A$ sufficiently large. Since the right-hand side of (14) is decreasing in $u_{0}$, it follows that $u_{0} \leq A(|x|+t)$. By a similar argument the solution of (14) exists and is unique.

Write $u_{0}(H, t)=u_{0}(\exp H, t)$. If $u_{0}=u_{0}(H, t)$ is a solution of (14) then for $a>0$ the solution of (14) for $a H$ and at is $a u_{0}$, so $a u_{0}=u_{0}(a H, a t)$. Thus $u_{0}(H, t)$ is 1-homogeneous. The homogeneity of $f\left(u_{0}\right)$ is now evident by (11).

We now give the exact bounds of the $\alpha$-stable density $p_{t}(x)$ in terms of $u_{0}$.

\section{Theorem 4.3.}

$$
p_{t}(x) \asymp \begin{cases}t\left(t^{1 / \alpha}+|x|\right)^{-n-\alpha} & \text { if } t+|x| \leqslant 1, \\ \phi_{0}(x) t^{\frac{1}{2-\alpha}}(|x|+t)^{-r(\alpha)+\frac{1}{2}} e^{-f\left(u_{0}\right)} & \text { if } t+|x| \geqslant 1 \text { and } u_{0} \leqslant t^{2 / \alpha}, \\ \phi_{0}(x) t(|x|+t)^{-s(\alpha)+\frac{1}{2}} e^{-f\left(u_{0}\right)} & \text { if } t+|x| \geqslant 1 \text { and } u_{0} \geqslant t^{2 / \alpha},\end{cases}
$$


with $r(\alpha)=\frac{l}{2}+\left|\Sigma^{++}\right|+\frac{4-\alpha}{4-2 \alpha}, s(\alpha)=\frac{l}{2}+\left|\Sigma^{++}\right|+1+\frac{\alpha}{2}, u_{0}=u_{0}(x, t)$ given implicitly by (13) and the function $f$ defined in (11).

Remark 1. Theorem 4.3 gives a weak scaling property for stable densities on symmetric spaces (the possibility of recovering, at least asymptotically, the density $p_{t}(x)$ if $p_{1}(x)$ is known).

More precisely, when $t+|x| \leqslant 1$, then $p_{t}(\exp H) \asymp t^{-n / \alpha} p_{1}\left(\exp \left(t^{-1 / \alpha} H\right)\right)$ (in the Euclidean case we have equality).

When $t+|x| \geqslant 1$, it is not the function $p_{t}(x)$ itself but the function $\phi_{0}(x)^{-1} p_{t}(x)$ that has a weak scaling property (the role of the function $\phi_{0}$ may be explained as the influence of the non-Euclidean structure of $X)$. It is clear that the factors $t^{\frac{1}{2-\alpha}}(|H|+t)^{-r(\alpha)+\frac{1}{2}}$ and $t(|H|+t)^{-s(\alpha)+\frac{1}{2}}$ of the bounds of the function $\phi_{0}(x)^{-1} p_{t}(\exp H)$ may be obtained from the factors $(|H|+1)^{-r(\alpha)+\frac{1}{2}}$ and $(|H|+1)^{-s(\alpha)+\frac{1}{2}}$ of the bounds of $\phi_{0}(x)^{-1} p_{1}(\exp H)$ by transformations of the form $H \mapsto t^{\beta} p_{1}\left(\exp \left(t^{-1} H\right)\right)$, with a convenient value of $\beta$. The same is true for the function $f\left(u_{0}\right)$ since, by the 1-homogeneity of $f\left(u_{0}\right)$, we have $t f\left(u_{0}\left(\exp \left(t^{-1} H, 1\right)=f\left(u_{0}(\exp H, t)\right)\right.\right.$.

On structures different from $\mathbb{R}$ stable measures defined by subordination may preserve such a weaker scaling property (cf. [BSS] for fractals). We answer in this way a question raised by Getoor in [Ge] about looking for "stability" properties of the densities $p_{t}$.

Remark 2. After the proof of the theorem we will give a simple criterion concerning the conditions $u_{0} \geqslant t^{2 / \alpha}$ and $u_{0} \leqslant t^{2 / \alpha}$. Note that for $u_{0} \asymp t^{2 / \alpha}$ the second and the third estimates coincide.

Proof of Theorem 4.3. The estimates for small $t$ and $|x|$ are the same as on $\mathbb{R}^{n}$ and may be found in [BSS] (cf. also [Ben]). From Lemma 4.2 it follows that $u_{0}(x, t) \rightarrow \infty$ when $\max (x, t) \rightarrow \infty$. Following $[\mathbf{A J}]$, Theorem 4.3.1, we will look for the estimates of $p_{t}(x)$ in terms of $u_{0}$, when $u_{0} \rightarrow \infty$. We split the integral (3) for $p_{t}(x)$ into

$$
\int_{0}^{\kappa^{-1} u_{0}}+\int_{\kappa^{-1} u_{0}}^{\infty}=I_{1}+I_{2}
$$

for a constant $\kappa>1$. We will see below that the first integral is essentially smaller than the second one, so $p_{t}(x) \asymp \int_{\kappa^{-1} u_{0}}^{\infty} h_{u}(x) \eta_{t}(u) d u$. Now we may use the estimate for $h_{u}(x)$ from the Theorem 2.1. Write $J_{2}=\phi_{0}(x)^{-1} I_{2}$ and in $J_{2}$ apply the change of variables $u=u_{0}(x, t) v$. Set

$$
P=P(x, t)=\frac{|x|^{2}}{4 u_{0}} /|\rho|^{2} u_{0} \quad \text { and } \quad Q=Q(x, t)=\frac{c_{1} \alpha}{2-\alpha} t^{\frac{2}{2-\alpha}} u_{0}^{\frac{-\alpha}{2-\alpha}} /|\rho|^{2} u_{0} .
$$

We have $0<P, Q<1$ and $P+Q \equiv 1$ (the functions $P$ and $Q$ measure in a sense the proportion of importance of variables $x$ and $t$ respectively in the 
function $\left.u_{0}(x, t)\right)$. We get

$$
f\left(u_{0} v\right)=|\rho|^{2} u_{0}\left(v+P \frac{1}{v}+Q \frac{2-\alpha}{\alpha} v^{-\frac{\alpha}{2-\alpha}}\right) .
$$

For any values of $P$ and $Q$, the point $v$ where the function

$$
g(v, x, t)=v+P \frac{1}{v}+Q \frac{2-\alpha}{\alpha} v^{-\frac{\alpha}{2-\alpha}}
$$

attains its minimum must satisfy the equation $P \frac{1}{v^{2}}+Q v^{-\frac{2}{2-\alpha}}=1$, which admits the only solution $v=1$. The functions $u_{0}, P$ and $Q$ depend on $x$ and $t$. Nevertheless, due to the uniqueness property just mentioned and the fact that $\frac{\partial^{2} g}{\partial v^{2}} \asymp 1$ for all $x$ and $t$, the proof of the Laplace method (see $[\mathbf{O}]$, pp. 80-82)) may be adapted to the present situation. The moderate price to pay is obtaining bounds instead of asymptotics in $u_{0}$. Consequently we get

$$
\begin{array}{ll}
J_{2} \asymp c t^{\frac{1}{2-\alpha}} u_{0}^{-r(\alpha)+\frac{1}{2}} e^{-f\left(u_{0}\right)}, \quad u_{0} \rightarrow \infty, & u_{0} \leqslant t^{2 / \alpha}, \\
J_{2} \asymp c t u_{0}^{-s(\alpha)+\frac{1}{2}} e^{-f\left(u_{0}\right)}, \quad u_{0} \rightarrow \infty, & u_{0} \geqslant t^{2 / \alpha} .
\end{array}
$$

To complete the proof we need to justify the claim that the first integral in (15), which we denote by $I_{1}$, is essentially smaller that the second one. We use the global upper estimate for the heat kernel ([An], [AJ, (3.3)]), and proceed as with $J_{2}$ above, applying the Laplace method. It follows that $I_{1} / I_{2} \rightarrow 0$ when $u_{0} \rightarrow \infty$.

Lemma 4.4. There exist positive constants $K, M$ such that if $t \leqslant K|x|^{\alpha / 2}$ then $u_{0} \geqslant t^{2 / \alpha}$ and if $t \geqslant M|x|^{\alpha / 2}$ then $u_{0} \leqslant t^{2 / \alpha}$. If $t \asymp|x|^{\alpha / 2}$ then $u_{0} \asymp t^{2 / \alpha}$.

Proof. The first two assertions follow easily from Lemma 4.2. If $t \asymp|x|^{\alpha / 2}$ then $|x| \asymp t^{2 / \alpha}$ and, consequently, $u_{0} \asymp|x|+t \asymp t^{2 / \alpha}+t$. Since $u_{0}$ and $t$ are supposed to be large $(t \rightarrow \infty)$, we have $u_{0} \asymp t^{2 / \alpha}$.

\section{Properties of $u_{0}$}

Note that for general $\alpha \in(0,2)$ we have no explicit formula for $u_{0}$ and $f\left(u_{0}\right)$. In this section we study their properties in greater depth.

Proposition 5.1. $(H, t) \mapsto u_{0}=u_{0}(\exp H, t)$ is a norm on $\mathfrak{a} \times \mathbb{R}^{+}$.

Proof. Again set $u_{0}(H, t)=u_{0}(\exp H, t)$. We already know that $u_{0}(H, t)$ is 1-homogeneous. Set $u_{0}=u_{0}(H, t), u_{1}=u_{0}\left(H_{1}, t_{1}\right), u_{2}=u_{0}\left(H+H_{1}, t+t_{1}\right)$, $a=1 /(4|\rho|)^{2}$ and $b=c_{2} /|\rho|^{2}$. For $p=\frac{u_{0}}{u_{0}+u_{1}} \in(0,1)$ and $q=1-p$ we have

$$
\frac{u_{0}}{p}=\frac{u_{1}}{q} .
$$


Then we obtain

$$
a p\left(\frac{|H|}{u_{0}}\right)^{2}+b p\left(\frac{t}{u_{0}}\right)^{\frac{2}{2-\alpha}}=p, \quad \text { and } \quad a q\left(\frac{\left|H_{1}\right|}{u_{1}}\right)^{2}+b q\left(\frac{t_{1}}{u_{1}}\right)^{\frac{2}{2-\alpha}}=q .
$$

Summing these equations, using the fact that functions $x \rightarrow x^{2}$ and $t \rightarrow$ $t^{2 /(2-\alpha)}$ are convex, and applying (19), we get $u_{2} \leq u_{0} / p=u_{1} / q$ and $u_{2} \leq$ $u_{0}+u_{1}$. This shows that $u_{0}$ is a norm.

Proposition 5.2. $u_{0}$ extends to a norm on $\mathfrak{a} \times \mathbb{R} . \quad f\left(u_{0}\right)$ is a norm on $\mathfrak{a} \times \mathbb{R}^{+}$and extends to a norm on $\mathfrak{a} \times \mathbb{R}$.

Proof. Since $u_{0}$ is homogeneous, it is determined by its unit sphere $S=$ $\left\{(H, t): u_{0}(H, t)=1\right\}$. Since $c_{2}^{(2-\alpha) / 2}=\frac{\alpha}{2}, S$ can be described by

$$
t=\frac{2}{\alpha}\left(|\rho|^{2}-\frac{|H|^{2}}{4}\right)^{\frac{2-\alpha}{2}} .
$$

One may deduce from this formula the convexity of the unit ball $B_{0} \subset \mathbb{R}^{2}$ of the 1-homogeneous function

$$
U_{0}: U_{0}(y, t)=u_{0}(|H|,|t|)
$$

with any $H \in \mathfrak{a}$ such that $|y|=|H|, y \in \mathbb{R}$. Thus $U_{0}$ is a norm on $\mathbb{R}^{2}$. The convexity of $B_{0}$ implies the convexity of

$$
B=\left\{(H, t) \in \mathfrak{a} \times \mathbb{R}:(|H|, t) \in B_{0}\right\}=\left\{(H, t) \in \mathfrak{a} \times \mathbb{R}: u_{0}(H,|t|)=1\right\},
$$

which is the unit ball of $u_{0}$ extended naturally to $\mathfrak{a} \times \mathbb{R}$. This gives the first statement.

For the second one, we solve $f\left(u_{0}\right)=1$ using (11) and the equality

$$
\left(\frac{t}{u_{0}}\right)^{\frac{2}{2-\alpha}}=\frac{1}{c_{2}}\left(|\rho|^{2}-\frac{1}{4}\left(\frac{|H|}{u_{0}}\right)^{2}\right) .
$$

We obtain a quadratic equation for $u_{0}$,

$$
\frac{2}{\alpha}|\rho|^{2} u_{0}^{2}-u_{0}+\frac{\alpha-1}{2 \alpha}|H|^{2}=0,
$$

and it follows that

$$
u_{0}=\varphi(H)=\frac{\alpha}{4|\rho|^{2}}(1+P(H)), \quad|H| \in\left(0, \frac{1}{|\rho|}\right),
$$

where

$$
P(H)=\sqrt{1-\frac{4(\alpha-1)}{\alpha^{2}}|\rho|^{2}|H|^{2}} .
$$

Indeed, let $\varphi_{m}(H)$ be the alternative formal solution to (21) (this is possible at least for $\alpha>1$ ). Then positivity of the right-hand side of (20) implies that $\frac{|H|}{2 \varphi_{m}(H)} \leq|\rho|$ and, consequently, $P(H) \leq 1-\frac{2|H||\rho|}{\alpha}$. This is possible only for $|H| \leq \frac{\alpha}{2|\rho|}$. Solving this we obtain $|H| \geq \frac{1}{|\rho|}$. The contradiction 
shows that (21) admits at most one solution. An analogous argument for $\varphi$ instead of $\varphi_{m}$ establishes the range for $H$. Note that for $|H| \in(0,1 /|\rho|)$ the determinant of $(21)$ is positive for every $\alpha \in(0,2)$. Thus, we arrive at $(22)$. Putting $u_{0}=\varphi(H)$ into (20) we get

$$
t=\psi(H)=\frac{2}{\alpha} \varphi(H)\left(|\rho|^{2}-\frac{|H|^{2}}{4 \varphi(H)^{2}}\right)^{1-\frac{\alpha}{2}} .
$$

We have $f\left(u_{0}\right)=1$ iff $u_{0}=\varphi(H)$ and $t=\psi(H)$. To finish the proof it is enough to show that the graph of $y \mapsto \psi(y)$ is concave on $\mathbb{R}^{+}$(i.e., $\psi^{\prime \prime} \leq 0$, where we understand $y=|H|$ and $\psi$ as a function of $|H|$ on $\mathbb{R}^{+}$) and the symmetry with respect to the $t$-axis does not affect the concavity (e.g., $\psi$ is decreasing). We obtain

$$
\psi^{\prime \prime}(y)=-\left(\frac{\alpha}{2}\right)^{\frac{\alpha}{2}} \frac{2 r^{2-\alpha}(1+P(y))^{\alpha}}{s(y)^{1+\frac{\alpha}{2}} P(y)}
$$

with the obvious meaning of $P(y)$ and

$$
s(y)=\alpha-2|\rho|^{2}|y|^{2}+\sqrt{\alpha^{2}-4(\alpha-1)|\rho|^{2}|y|^{2}}>0,
$$

since for $y<1 /|\rho|$ we have

$$
s(y) \geq \alpha-2+\sqrt{\alpha^{2}-4(\alpha-1)}=\alpha-2+|\alpha-2|=0 .
$$

These formulas can be quickly checked using Maple or Mathematica. Thus $\psi^{\prime \prime}(y) \leq 0$. It is easy to verify that $\psi^{\prime}(0)=0$, so $\psi^{\prime}(y) \leq 0$ as required.

Corollary 5.3. When $t+|x| \geqslant 1$,

$$
p_{t}(x) \asymp \begin{cases}\phi_{0}(x) t^{\frac{1}{2-\alpha}}(|x|+t)^{-r(\alpha)+\frac{1}{2}} e^{\sqrt{|x|^{2}+t^{2}} q_{\alpha}} & \text { if } u_{0} \leqslant t^{2 / \alpha}, \\ \phi_{0}(x) t(|x|+t)^{-s(\alpha)+\frac{1}{2}} e^{\sqrt{|x|^{2}+t^{2}} q_{\alpha}} & \text { if } u_{0} \geqslant t^{2 / \alpha},\end{cases}
$$

where the function $q_{\alpha}$ satisfies

$$
q_{\alpha}\left(k \exp H k^{\prime}, t\right)=q_{\alpha}\left(\frac{(H, t)}{\|(H, t)\|_{2}}\right), \quad k, k^{\prime} \in K,
$$

and is a continuous, bounded and bounded away from zero function on the Euclidean unit sphere in $\mathfrak{a} \times \mathbb{R}$.

Proof. Set $q_{\alpha}(\exp H, t)=f\left(u_{0}(\exp H, t)\right) / \sqrt{|H|^{2}+t^{2}}$. By Proposition 5.3 the function $(H, t) \rightarrow q_{\alpha}(\exp H, t)$ is homogeneous of degree 0 .

Remark. When $\alpha=1$ the function $q_{\alpha}$ is constant. The bounds from the Corollary 5.3 are an extension of the bounds obtained in $[\mathbf{A J}]$ for $\alpha=1$.

Lemma 4.2 gives enough information to replace $u_{0}$ in an estimate when it is a multiplicative factor. To deal with the exponent $e^{-f\left(u_{0}\right)}$ in the estimates of $p_{t}(x)$ more delicate (additive) properties are required. Namely,

$$
\exp (f(x)) \asymp \exp (g(x)) \Longleftrightarrow|f-g| \text { is bounded. }
$$


This motivates the following definition: we write

$$
f(x) \stackrel{e}{\asymp} g(x)
$$

if there exists $M \in \mathbb{R}$ such that $|f(x)-g(x)| \leq M$ for all $x$.

Proposition 5.4. If $t \leqslant|x|^{\alpha / 2}$ then

$$
\exp \left(-f\left(u_{0}\right)\right) \asymp \exp (-|\rho||x|) .
$$

In particular, this holds for fixed $t>0$, as $x \rightarrow \infty$ (that is, for all sufficiently large $\left.x>x_{0}(t)\right)$. Moreover, if $t=|x|^{s}$ with $s>\alpha / 2$ then (23) does not hold.

Proof. By our assumption, $t^{\frac{2}{2-\alpha}} \leq|x|^{\frac{\alpha}{2-\alpha}}$ and

$$
t^{\frac{2}{2-\alpha}} u_{0}^{-\frac{\alpha}{2-\alpha}} \leq\left(\frac{|x|}{u_{0}}\right)^{\frac{\alpha}{2-\alpha}}
$$

The right-hand side of this inequality is bounded since $u_{0} \asymp|x|+t$. Thus, Equation (14) multiplied by $u_{0}$ gives

$$
f\left(u_{0}\right)=\frac{|x|^{2}}{4 u_{0}}+|\rho|^{2} u_{0}+c_{1} t^{\frac{2}{2-\alpha}} u_{0}^{-\frac{\alpha}{2-\alpha}} \stackrel{e}{=} 2|\rho|^{2} u_{0} .
$$

Using (13) and (24) we deduce that $u_{0} \stackrel{e}{`} \frac{|x|}{2|\rho|}$, which together with (25) gives the first assertion.

By the definition of $f\left(u_{0}\right)$ for $t=|x|^{s}$ and by (13) we obtain

$$
f\left(u_{0}\right)-|\rho||x|=\left(c_{1}+c_{2}\right)\left(\frac{|x|}{u_{0}}\right)^{\frac{\alpha}{2-\alpha}}|x|^{\frac{2 s-\alpha}{2-\alpha}}-|x|\left(|\rho|-\frac{|x|}{2 u_{0}}\right) .
$$

By (14)

$$
\left(|\rho|-\frac{|x|}{2 u_{0}}\right)\left(|\rho|+\frac{|x|}{2 u_{0}}\right)=c_{2}\left(\frac{t}{u_{0}}\right)^{\frac{2}{2-\alpha}}
$$

so, transforming (26) with $t=|x|^{s}$ we get

$$
\begin{aligned}
& f\left(u_{0}\right)-|\rho||x| \\
& =|x|^{\frac{2 s-\alpha}{2-\alpha}}\left(\left(c_{1}+c_{2}\right)\left(\frac{|x|}{u_{0}}\right)^{\frac{\alpha}{2-\alpha}}-c_{2}\left(\frac{|x|}{u_{0}}\right)^{\frac{2}{2-\alpha}}\left(|\rho|+\frac{|x|}{2 u_{0}}\right)^{-1}\right) .
\end{aligned}
$$

Note that for $s \leq \alpha / 2$ this is bounded (for $s<\alpha / 2$ it even tends to 0 ), since $u_{0} \asymp|x|+t$. (This gives once again the first assertion.) From (14) with $|x|=t^{1 / s}$ we deduce that $\frac{t}{u_{0}} \rightarrow 0$. Observe that it is enough to show the second assertion for $s \in(\alpha / 2,1)$.

Now, from (14) it follows that

$$
\frac{|x|}{2 u_{0}} \rightarrow|\rho|
$$


Together with (28) it implies that for $s>\alpha / 2$ we have $f\left(u_{0}\right)-|\rho||x| \rightarrow \infty$, which completes the proof.

Proposition 5.5. If $|x| \leqslant \sqrt{t}$ then $\exp \left(-f\left(u_{0}\right)\right) \asymp \exp \left(-|\rho|^{\alpha} t\right)$. This is true, in particular, when $x$ is fixed and $t \rightarrow \infty$ (that is, for all sufficiently large $\left.t>t_{0}(x)\right)$. If $|x|=t^{s}$ with $s>\frac{1}{2}$, it is no longer true.

Proof. Multiplying (14) by $u_{0}$ and using

$$
\frac{|x|^{2}}{u_{0}} \leq \frac{t}{u_{0}}
$$

which is bounded since $u_{0} \asymp|x|+t$, we get

$$
f\left(u_{0}\right) \stackrel{e}{\asymp}|\rho|^{2} u_{0}+\frac{2-\alpha}{\alpha}|\rho|^{2} u_{0}=\frac{2}{\alpha}|\rho|^{2} u_{0} .
$$

We claim that

$$
\frac{2}{\alpha}|\rho|^{2} u_{0} \stackrel{e}{\asymp}|\rho|^{\alpha} t
$$

We will use the following simple consequence of the Mean Value Theorem: for $0<a<b$ and any $\gamma$ we have

$$
b^{\gamma}-a^{\gamma}=\gamma K^{\gamma-1}(b-a),
$$

for some $K \in(a, b)$. From (14) multiplied by $u_{0}^{2 /(2-\alpha)}$ we have

$$
|\rho|^{2} u_{0}^{\frac{2}{2-\alpha}}-c_{2} t^{\frac{2}{2-\alpha}}=\frac{|x|^{2}}{4} u_{0}^{-2+\frac{2}{2-\alpha}} .
$$

From this and (33) applied to $a=c_{2} t^{\frac{2}{2-\alpha}}, b=|\rho|^{2} u_{0}^{\frac{2}{2-\alpha}}$ and $\gamma=\frac{2-\alpha}{2}$ we get

$$
|\rho|^{2-\alpha} u_{0}-c_{2}^{\frac{2-\alpha}{2}} t=\frac{2-\alpha}{2} K^{-\frac{\alpha}{2}} \frac{|x|^{2}}{4} u_{0}^{-2+\frac{2}{2-\alpha}},
$$

with some $K \in(a, b)$. Consequently, as $K>a$,

$$
|\rho|^{2-\alpha} u_{0}-c_{2}^{\frac{2-\alpha}{2}} t \leq c\left(\frac{u_{0}}{t}\right)^{\frac{\alpha}{2-\alpha}} \frac{|x|^{2}}{u_{0}}
$$

which is bounded by virtue of (30) and the fact that $|x|^{2} \leq t$ yields $u_{0} \asymp$ $|x|+t \asymp t$. It follows that

$$
|\rho|^{2-\alpha} u_{0} \stackrel{e}{\asymp} c_{2}^{\frac{2-\alpha}{2}} t
$$

To get our claim, multiply (34) by $\frac{2|\rho|^{\alpha}}{\alpha}$ and use the fact that $\frac{2}{\alpha} c_{2}^{\frac{2-\alpha}{2}}=1$. The first assertion now follows from (31) and (32). 
In order to prove the second assertion, we show in a way similar to the proof of the second assertion of the Proposition 5.4 (but using the Mean Value Theorem as above, for $\gamma=\alpha / 2$ ) that the formula

$$
f\left(u_{0}\right)-|\rho|^{\alpha} t=\frac{1}{2} t^{2 s-1}\left(\frac{t}{u_{0}}-\frac{\alpha}{4}\left(\frac{t}{u_{0}}\right)^{2} K^{\frac{\alpha-2}{2}}\right)
$$

holds when $|x|=t^{s}$, with

$$
c_{2}\left(\frac{t}{u_{0}}\right)^{\frac{2}{2-\alpha}}<K<|\rho|^{2} .
$$

The case $s>1$ being evident by (14), we see that for $s<1$, again using (14), we have $\frac{|x|}{u_{0}} \rightarrow 0$ and

$$
\frac{t}{u_{0}} \rightarrow c_{2}^{\frac{\alpha-2}{2}}|\rho|^{2-\alpha}
$$

when $|x|=t^{s}$.

Taking limits in (36) we get $K \rightarrow|\rho|^{2}$, so $K^{\frac{\alpha-2}{2}} \rightarrow|\rho|^{\alpha-2}$. Thus the expression between the brackets in (35) does not tend to 0 . It follows that for $s>1 / 2$ the difference $f\left(u_{0}\right)-|\rho|^{\alpha} t$ is unbounded when $t \rightarrow \infty$ and $|x|=t^{s}$.

Corollary 5.6. For any constants $K, M>0$ we have $p_{t}(x) \asymp \begin{cases}\phi_{0}(x) t^{\frac{1}{2-\alpha}}(|x|+t)^{-r(\alpha)+\frac{1}{2}} e^{-|\rho|^{\alpha} t} & \text { if } t+|x| \geqslant 1 \text { and }|x| \leqslant K t^{1 / 2}, \\ \phi_{0}(x) t(|x|+t)^{-s(\alpha)+\frac{1}{2}} e^{-|\rho||x|} & \text { if } t+|x| \geqslant 1 \text { and }|x| \geqslant M t^{2 / \alpha} .\end{cases}$

On the other hand, in a region $K t^{1 / 2}<|x|<M t^{2 / \alpha}$ one cannot give a simpler estimate of $e^{-f\left(u_{0}\right)}$ and, consequently, a simpler estimate of $p_{t}(x)$ than the one given in the Theorem 4.3 .

Proof. Only the last assertion needs to be justified. Suppose that $g(|x|, t)$ is a 1-homogeneous function such that $e^{-f\left(u_{0}\right)} \asymp e^{-g}$ on

$$
R=\left\{(|x|, t): K t^{1 / 2}<|x|<M t^{2 / \alpha}\right\} .
$$

It implies that $f\left(u_{0}\right) \stackrel{e}{\asymp} g$.

Observe that the region $R$ contains half-lines of the form $|x|=a t$, where $t>t_{0}(a), a>0$. The function $f\left(u_{0}\right)-g$ is homogeneous of degree 1 and bounded on $R$, so $f\left(u_{0}\right)=g$ on $R$.

Suppose that the function $f\left(u_{0}\right)$ is explicitly determined. Writing (11) in the form

$$
f\left(u_{0}\right)=\frac{|x|^{2}}{4 u_{0}}+|\rho|^{2} u_{0}+c_{1} u_{0}\left(\frac{t}{u_{0}}\right)^{\frac{2}{2-\alpha}}
$$


and replacing $\left(\frac{t}{u_{0}}\right)^{\frac{2}{2-\alpha}}$ by

$$
c_{2}^{-1}\left(|\rho|^{2}-\frac{1}{4}\left(\frac{|x|}{u_{0}}\right)^{2}\right)
$$

we get according to (14) a quadratic equation with respect to $u_{0}$ that could be solved explicitly. Except for some special values of $\alpha=1, \frac{3}{2}, \ldots$, this is not possible.

Acknowledgements. We thank J.-P. Anker, K. Bogdan and T. Byczkowski for discussions and helpful comments on the subject of the paper. The second named author acknowledges the hospitality of Université d'Angers during a post-doctoral fellowship granted by the region Pays de la Loire.

\section{References}

[An] J.-P. Anker, Sharp estimates for some functions of the Laplacian on noncompact symmetric spaces, Duke Math. J., 65 (1992), 257-297, MR 1150587 (93b:43007), Zbl 0764.43005.

[AJ] J.-P. Anker and L. Ji, Heat kernel and Green function estimates on noncompact symmetric spaces, Geom. Funct. Anal., 9(6) (1999), 1035-1091, MR 1736928 (2001b:58038), Zbl 0942.43005.

[App1] D. Applebaum, Lévy processes in stochastic differential geometry, Lévy processes, 111-137, Birkhäuser, Boston, MA, 2001, MR 1833695 (2002d:60038), Zbl 0984.60056.

[App2] D. Applebaum, On the subordination of spherically symmetric Lévy processes in Lie groups, Int. Math. J., 1(2) (2002), 185-194, MR 1828652 (2002f:60011), Zbl 0984.60018.

[Ben] A. Bendikov, Asymptotic formulas for symmetric stable semigroups, Exposition. Math., 12 (1994), 381-384, MR 1297844 (95j:60029), Zbl 0810.60070.

[Be] J. Bertoin, Lévy Processes, Cambridge Tracts in Mathematics, 121, Cambridge University Press, Cambridge, 1996, MR 1406564 (98e:60117), Zbl 0861.60003.

[BG] R.M. Blumenthal and R.K. Getoor, Markov Processes and Potential Theory, Pure and Applied Mathematics, 29, Academic Press, New York, 1968, MR 0264757 (41 \#9348), Zbl 0169.49204.

[BSS] K. Bogdan, A. Stós and P. Sztonyk, Harnack inequality for symmetric stable processes on fractals, C.R. Acad. Sci. Paris, Ser. I, 335(1) (2002), 59-63, MR 1920996 (2003k:60100), Zbl 1019.60078.

[D] G. Doetsch, Introduction to the Theory and Application of the Laplace Transformation, Springer, Berlin-Heidelberg-New York, 1974, MR 0344810 (49 \#9549), Zbl 0278.44001.

[Ge] R.K. Getoor, Infinitely divisible probabilities on the hyperbolic plane, Pacific J. Math., 11 (1961), 1287-1308, MR 0133858 (24 \#A3682), Zbl 0124.34502.

[GR] I.S. Gradshteyn and I.M. Ryzhik, Table of Integrals, Series and Products, 6th edition, Academic Press, London, 1965, MR 0197789 (33 \#5952), Zbl 0981.65001. 
[Gruet] J-C. Gruet, Jacobi radial stable processes, Ann. Math. Blaise Pascal, 5 (1998), 39-48, MR 1671691 (99m:60015), Zbl 0926.60020.

$[\mathrm{H}] \quad$ J. Hawkes, A lower Lipschitz condition for the stable subordinator, Z. Wahrscheinlichkeitstheorie und Verw. Gebiete, 17 (1971), 23-32, MR 0282413 (43 \#8125), Zbl 0193.45002.

[Ku] H. Kunita, Stable Lévy processes on nilpotent Lie groups, in 'Stochastic analysis on infinite-dimensional spaces' (Baton Rouge, LA, 1994), 167-182, Pitman Res. Notes Math. Ser., 310, Longman Sci. Tech., Harlow, 1994, MR 1415667 (97k:60019), Zbl 0814.60003.

[O] F.W.J. Olver, Asymptotics and Special Functions, Computer Science and Applied Mathematics, Academic Press, New York-London, 1974, MR 0435697 (55 \#8655), Zbl 0303.41035.

Received December 3, 2002 and revised January 14, 2004. Both authors supported by KBN grant 2 P03A 04122 and RTN Harmonic Analysis and Related Problems contract HPRN-CT-2001-00273-HARP.

DÉPARTEMENT DE MATHÉMATIQUeS

UNIVERSITÉ D'ANGERS

FRANCE

E-mail address: graczyk@univ-angers.fr

Institute OF MATHEMATICS

WrocŁaW UNIVERSITY OF TECHNOLOGY

WROCEAW

POLAND

E-mail address: stos@im.pwr.wroc.pl

LABoratoire de Mathématiques UMR 6620

Université Blaise Pascal

FRANCE 